\title{
Coordinated analysis of space weathering characteristics in lunar samples to understand water distribution on the Moon
}

\author{
ALEXANDER M KLING ${ }^{1}$, MICHELLE S. THOMPSON ${ }^{1}$, \\ JENNIKA GREER ${ }^{2,3}$ AND PHILIPP R HECK ${ }^{2,4}$ \\ ${ }^{1}$ Purdue University \\ ${ }^{2}$ University of Chicago \\ ${ }^{3}$ The Field Museum \\ ${ }^{4}$ The Field Museum of Natural History \\ Presenting Author: klinga@purdue.edu
}

Space weathering, driven by solar wind irradiation and micrometeorite bombardment, alters the optical, chemical, and microstructural properties of grains on the surfaces of airless bodies [1]. Microstructural changes in the outermost rims (100 $\mathrm{nm}$ ) of space weathered grains include vapor and melt deposits formed by micrometeorite impacts and amorphous rims formed by solar wind irradiation. These rims may contain nanoparticles composed of reduced Fe metal, called nanophase iron (npFe), and vesicles that may be formed by the coalescence of implanted solar wind $\mathrm{H}$ and $\mathrm{He}$ [2]. Previous work has shown that space weathered rims of interplanetary dust particles (IDPs) can also contain vesicles and amorphous rims formed by solar wind irradiation. Valence electron energy loss spectroscopy (VEELS) measurements of these IDPs revealed that the vesicles and amorphous rims contain water in both the $\mathrm{H}_{2} \mathrm{O}$ and $\mathrm{OH}$ molecular forms [3]. However, the precise nature of the relationship between space weathering and the formation and retention of water within space weathered rims is still unknown. We present preliminary analyses of the space weathered rims of lunar soil particles with plans to conduct a series of coordinated analyses including atom probe tomography (APT) and transmission electron microscopy (TEM) analyses to better characterize the relationship between water and space weathering characteristics.

We used focused ion beam scanning electron microscopy (FIB-SEM) to prepare an electron-transparent section of a lunar soil grain that displayed evidence of a solar wind irradiated rim for TEM analysis. We then performed high resolution TEM (HRTEM) imaging of the rim to characterize evidence of space weathering and are currently performing chemical mapping. After initial characterization, we will perform APT of a region adjacent to where the TEM FIB section was extracted to ensure a likelihood of continuity between the microstructure of the two regions to correlate TEM and APT measurements.

[1] Pieters \& Noble (2016) JGR: Planets, 121(10), 18651884. [2] Keller \& McKay (1997). Geochim. Cosmochim. Acta, 61, 2311-2341. [3] Bradley, J. P. et al. (2014). PNAS, 111(5), $1732-1735$. 